\title{
Sopi or not sopi ?
}

A propos des élections présidentielles de février 2007 au Sénégal

\section{Géraud Magrin}

\section{OpenEdition}

\section{Journals}

Édition électronique

URL : http://journals.openedition.org/echogeo/838

DOI : $10.4000 /$ echogeo.838

ISSN : 1963-1197

\section{Éditeur}

Pôle de recherche pour l'organisation et la diffusion de l'information géographique (CNRS UMR 8586)

\section{Référence électronique}

Géraud Magrin, «Sopi or not sopi ? », EchoGéo [En ligne], Sur le Vif, mis en ligne le 20 juin 2007, consulté le 19 avril 2019. URL : http://journals.openedition.org/echogeo/838 ; DOI : 10.4000/ echogeo.838

Ce document a été généré automatiquement le 19 avril 2019

\section{(c) (i) (3)}

EchoGéo est mis à disposition selon les termes de la licence Creative Commons Attribution - Pas d'Utilisation Commerciale - Pas de Modification 4.0 International 


\title{
Sopi ${ }^{1}$ or not sopi ?
}

\author{
A propos des élections présidentielles de février 2007 au Sénégal
}

\section{Géraud Magrin}

Introduction

1 Lors des élections sénégalaises du 25 février 2007, le président sortant, Abdoulaye Wade, a été réélu dès le premier tour avec près de $57 \%$ des voix ${ }^{2}$. Ce fut une surprise, sauf peutêtre pour le président et quelques uns de ses partisans. La grande majorité des observateurs, qu'ils soient sénégalais ou étrangers ${ }^{3}$, annonçaient une élection très ouverte. En effet, l'alternance de mars 2000, qui avait vu l'arrivée au pouvoir d'Abdoulaye Wade après quatre décennies de règne du Parti socialiste (PS), s'était accompagnée d'un immense espoir de changement. En 2005-2006, l'essor des flux migratoires de clandestins sénégalais vers l'Europe, via les Canaries, signalait l'ampleur de la distance entre la rhétorique du pouvoir, qui présente un Sénégal émergent, et des réalités politiques, sociales et économiques moins flatteuses ${ }^{4}$ : tensions politiques, scandales politicofinanciers, escarmourches entre le pouvoir et les médias privés, crise de secteurs sensibles de l'économie comme l'agriculture ou l'industrie, embouteillages structurels à Dakar, délestages, inflation... à maints égards, le contexte semblait favorable au mécontentement populaire et à des joutes électorales serrées.

2 L'erreur de diagnostic n'illustrait-elle pas seulement, comme s'en délectait un partisan du camp vainqueur au lendemain du scrutin sur les ondes de Radio France Internationale, l'aveuglement propre aux intellectuels face à la clairvoyance du peuple ? Ou bien peut-on considérer aussi que le vote du 25 février a traduit le décalage entre les opinions volontiers contestataires - exprimées par les Sénégalais et leur vote, où le respect des principes de bonne gouvernance pèserait moins que l'animation du jeu clientéliste? Sans entrer dans les arcanes d'une vie politique nationale volontiers byzantine, il s'agit ici de proposer quelques éclairages sur une des victoires électorales les plus nettes de l'histoire politique récente en Afrique $^{5}$, en les reliant autant que possible à quelques données de la géographie du pays.

1. Les raisons du suspens

3 Les mutations de la société sénégalaise et les déceptions de l'alternance semblaient suffisantes sinon pour empêcher la réélection du président sortant, du moins pour la 
compliquer. En effet, comme ailleurs en Afrique, la démographie constitue au Sénégal un puissant facteur de changement. La refonte des fichiers électoraux dans la perspective des élections de $2007^{6}$ renouvelle près de la moitié du corps électoral : celui-ci passe de 2,7 millions d'électeurs en 2000 à 4,9 millions en 2007, sur une population totale de 11 millions d'habitants. Les jeunes fournissent naturellement l'essentiel des nouveaux électeurs. Parallèlement, le Sénégal s'urbanise rapidement - le taux d'urbanisation dépasse $50 \%$ en 2007, il était de $33 \%$ en 1988 et de moins de $20 \%$ en 1960. L'importance de l'émigration contribue aussi à relâcher l'emprise des encadrements traditionnels (Dahou, Foucher, 2004). Globalement plus jeune, plus urbain et plus éduqué que par le passé, l'électorat semble aussi moins sensible à l'influence des porteurs de voix, chefs de village, de quartier, élus locaux et marabouts, relais habituels du pouvoir en place dans le système politique sénégalais. Base de l'élection de Wade en 2000, le vote des jeunes urbains apparaissait plus incertain. Malgré les promesses du sopi, ne sont-ils pas massivement frappés par le sous-emploi chronique et ainsi bercés par les rêves migratoires?

4 L'alternance de 2000 a soulevé au Sénégal un grand espoir. Après 40 ans au pouvoir, le régime socialiste était devenu très impopulaire. Certes, il avait maintenu la paix et fait vivre un système politique pluraliste, sinon démocratique, ce qui n'était pas si fréquent dans cette partie du monde. Mais le vent mauvais des sécheresses des années 1970-1980, aux effets aggravés par l'ajustement structurel, a réduit les marges de manœuvre et entravé l'effort de développement. Pour les Sénégalais, et surtout pour les jeunes, ce régime est indissociable de la crise. Les turpitudes d'une classe politique partagée entre sens de l'Etat et stratégies d'enrichissement personnel finirent de discréditer les élites au pouvoir. Dans cette perspective, l'espoir associé au sopi était, certes, celui d'une vie meilleure. Mais il était aussi celui d'un changement de mœurs politiques, vers plus de démocratie et de transparence dans la gestion de la chose publique, moins de clientélisme, de politique politicienne et de magouilles.

5 Or, c'est sans doute dans cette perspective que le rêve de changement a le plus été déçu. Le régime de l'alternance s'est inscrit dans une remarquable continuité par rapport aux lignes de force du système politique sénégalais antérieur, en amplifiant même certains travers (voir Dahou, Foucher, 2004). Ainsi, jamais les liens entre le politique et le religieux n'avaient été aussi étroits, même s'ils se manifestent sous des formes complexes. Les démonstrations d'appartenance et de fidélité confrérique du président Wade auprès des marabouts de Touba ont dérangé nombre de Sénégalais non mourides. Plus largement, la vie politique a été marquée par une forte instabilité, à travers de fréquents changements de gouvernement, où la docilité envers un chef de l'Etat autoritaire apparaissait comme le meilleur gage de promotion. Pressé de mettre en œuvre ses innombrables projets, le président se méfie des services de l'Etat, contourne les ministères en multipliant les agences ad hoc - 27 dans le dernier gouvernement de novembre $2006^{7}$ - et assouplit le fonctionnement de l'administration en l'informalisant. La télévision nationale, qui se diffuse très largement dans les ménages sénégalais à la faveur de l'urbanisation et de l'électrification des bourgs et petites villes, affiche dans ses journaux une partialité confinant au culte de la personnalité, qui alimente les sarcasmes au sein des familles. On raille un président davantage préoccupé de construire son ego que le pays.

6 Les milieux du pouvoir, agités par la rivalité entre fidèles du Parti démocratique sénégalais (PDS) souvent peu expérimentés dans la gestion des affaires de l'Etat et «transhumants $»^{8}$ opportunistes, se distinguent aussi par des enrichissements très 
rapides. Ce climat délétère, dénoncé par des journalistes, comme Abdou Latif Coulibaly (Coulibaly A., 2003), aboutit à des menaces portées sur des membres de la société civile ou des responsables religieux, voire à des violences politiques, comme l'agression dont fut victime l'opposant Talla Sylla en octobre 2003. Les médias privés - presse et radio demeurent très libres - sont volontiers critiques envers le gouvernement, mais ils font aussi souvent l'objet de poursuites qui maintiennent la tension (Havard, 2004). Enfin, l'affaire des chantiers de Thiès, où des entraves aux règles de passation des marchés pour la fête de l'indépendance du 4 avril 2004 sont montées en épingle pour faire arrêter Idrissa Seck, dauphin trop pressé tombé en disgrâce, déplaît à l'opinion qui ne goûte guère ce genre d'arbitraire 9 . D'autant que les travaux ont donné des résultats appréciés : la promenade des Thiessois et le centre rénové font l'orgueil des habitants de la ville.

7 L'incertitude est pimentée par la ronde des acteurs politiques : nombre de barons du PS, comme Djibo Ka et Ousmane Ngom, ou d'anciens laudateurs d'Abdou Diouf, comme le professeur Iba Der Thiam, ont rejoint le PDS au pouvoir. Les luttes intestines redoublent au PDS à l'approche des élections - partiellement calmées par la décision prise en janvier de découpler législatives et présidentielles. Mais Idrissa Seck, qui en était un poids lourd, le quitte pour fonder sa propre formation. Le nombre de candidatures est plus élevé qu'à l'accoutumé, malgré l'augmentation de la caution à verser, laissant entrevoir une possible dispersion des votes : sur 15 candidats, 9 n'appartiennent qu'à de très petits partis parfois créés pour la circonstance, 7 sont de quasi-inconnus issus de la société civile. Comme lors des élections de 2000, les marabouts à la tête des principales confréries affichent leur neutralité dans l'élection. Mais certains d'entre eux, y compris parmi les mourides, n'hésitent pas à critiquer un président à qui ils reprochent notamment ses promesses non tenues (Michel S., 2007a).

8 Au-delà des vicissitudes du microcosme politique, les difficultés du pouvoir face aux enjeux du développement apparaissent comme des facteurs susceptibles de lui compliquer les élections. Le régime de l'alternance a fait bien des promesses, mais les plus ambitieuses - la plate-forme de Diamnadio ${ }^{10}$, le nouvel aéroport de Diass, l'autoroute à péage Dakar Diamnadio, une nouvelle capitale, etc. - tardent à se concrétiser. Pour le reste, la multiplication des chantiers à travers tout le pays - cases des tout petits, espaces jeunes, foyers des femmes, centres de formation des producteurs, centres de stockage des produits agricoles, etc. - semble dessiner un Sénégal Potemkine, où les façades modernes ne sont soutenues par aucune stratégie claire ni aucune dynamique économique et sociale convaincante. Pendant des mois, et parfois 2 ou 3 ans, les bâtiments achevés semblent attendre, vides, que l'approche des élections justifie leur inauguration ${ }^{11}$.

La dépendance envers l'agriculture pluviale arachidière - qui permet la survie de ruraux encore nombreux, mais n'a permis aucune dynamique de développement solide dans les zones productrices - et la hausse des cours du pétrole ont révélé la fragilité de la croissance qui, en recul à 2,8 \% en 2006, ne permet plus que de suivre le rythme de l'essor démographique (Bernard, 2007b). De 2005 à 2007, la crise des bons impayés liée à la libéralisation de la filière arachidière mécontente les campagnes. Le boom immobilier à Dakar, alimenté notamment par la diaspora, nourrit la hausse des prix du logement tout en révélant la profondeur des contrastes sociaux, que les palais des nouveaux riches des Almadies soulignent avec vigueur. L'afflux des jeunes et des ruraux sur le marché du travail urbain ne trouve d'autre exutoire que l'émigration, aux formes actuelles si dangereuses. ${ }^{12}$ 
10 Finalement, des résultats de sondages menés principalement dans les grandes villes du Sénégal de l'Ouest ou sur internet circulent plus ou moins sous le manteau: ils accréditent l'idée selon laquelle les quatre principaux candidats (A. Wade, I.Seck, O.T. Dieng et M. Niasse) se tiendraient dans une fourchette de 15 à $25 \%$. Mais leur fiabilité est sujette à caution, car on ne sait pas trop comment ils sont réalisés.

2. Le Sénégal en bleu

11 Le résultat des élections balaie les nuages de ces incertitudes. De la vague bleue - la couleur du PDS, le parti présidentiel - n'émergent que d'infimes îlots gagnés par l'opposition. Un tel vote a-t-il pu être sincère? Certains des candidats malheureux, surpris et vexés par l'ampleur de la défaite, ont brandi l'habituelle hypothèque de la fraude, tristement banale en terre africaine. Mais avec un manque d'argument et de conviction qui dit bien leur désarroi. Leurs partisans présents dans les bureaux de vote n'ont pas constaté d'irrégularités massives. Il ne leur restait plus alors qu'à dénoncer un complot ourdi ailleurs, en amont, par exemple du côté de l'informatisation des fichiers électoraux. Leur requête auprès de la Cour constitutionnelle n'a logiquement pas abouti. Le mythe du complot informatisé - entré dans la vie politique sénégalaise lorsque des CD de révélations compromettantes sur le président ont commencé à circuler sous le manteau lors de l'incarcération d'Idrissa Seck - n'a pas pris. Les observateurs électoraux nationaux et étrangers ont unanimement validé un scrutin que quelques irrégularités mineures n'ont pas dénaturé. Contre toute attente, le président Wade a bien été plébiscité par le plus grand nombre de Sénégalais ayant jamais pris part à une élection - le taux de participation dépassant $70 \%$.

\section{Score Abdoulaye Wade (pourcentage par Département)}

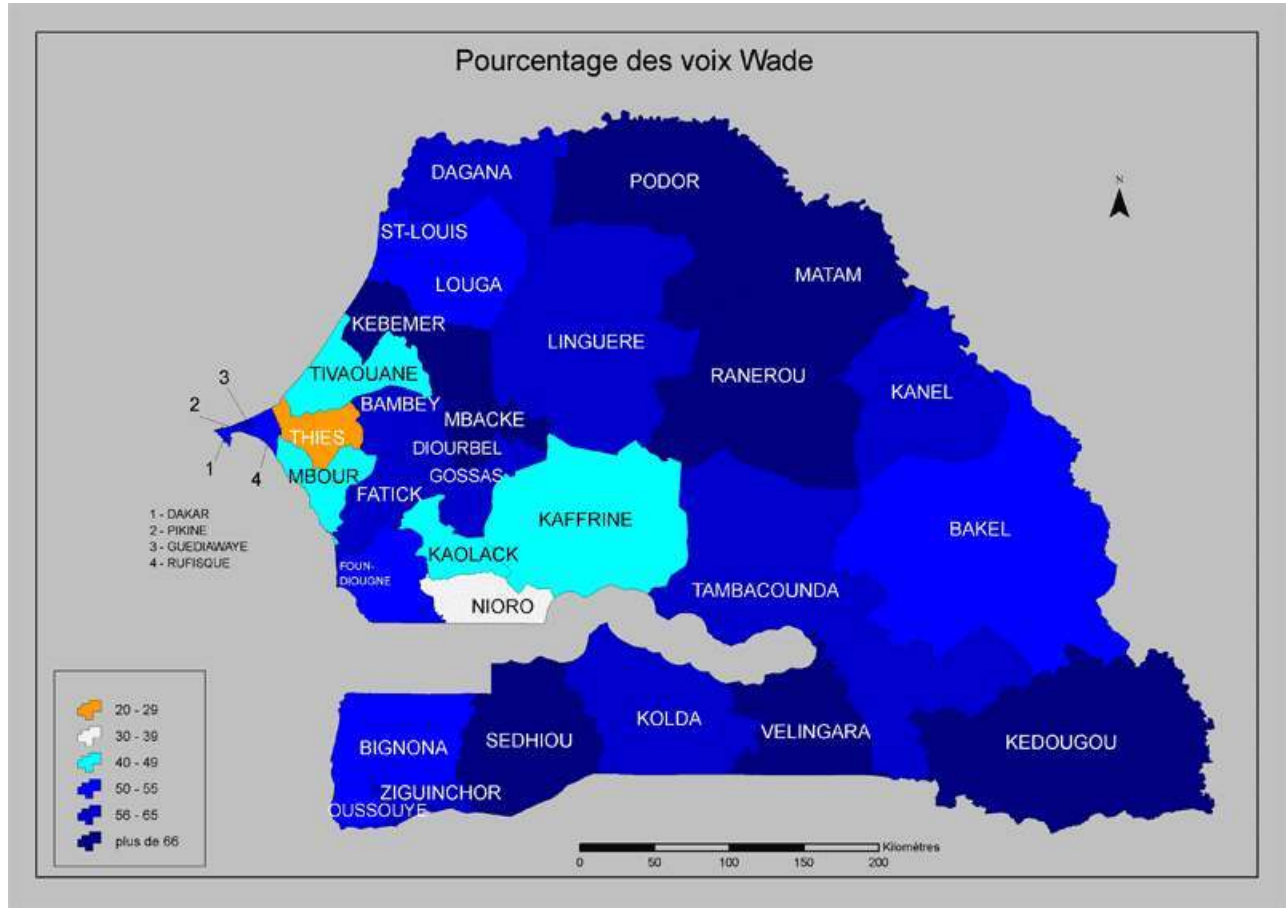

Source : les chiffres utilisés sont les résultats provisoires de la commission nationale de recensement des votes, Sud Quotidien, 2 mars 2007.

Le succès du sortant se donne à lire dans une carte électorale du Sénégal bleu Klein (cf. carte). Sur 35 départements, Wade arrive en tête partout, sauf à Thiès. Il atteint la 
majorité absolue de $50 \%$ dans 29 départements, dont les plus peuplés comme ceux de la Presqu'île du Cap Vert (Dakar et ses banlieues). Il dépasse sa moyenne nationale (55,86 \%) dans 20 départements, et est même littéralement plébiscité avec plus des deux tiers des suffrages dans 8 départements.

Ses scores les plus élevés relèvent de deux ensembles socio-spatiaux différents. Ils concernent le centre ouest et le nord du bassin arachidier (Baol, Cayor, Djolof), au peuplement majoritairement Wolof, où ses origines familiales - sa famille vient de Kébémer - mais surtout sa proximité affichée avec le mouridisme, lui valent des scores impressionnants (près de $84 \%$ dans le département de Mbacké, qui abrite Touba, capitale des Mourides).

Le ralliement massif des régions périphériques au président Wade constitue en revanche un fait nouveau. Certes, leur faible densité démographique (environ 5 habitants au km2) atténue le poids électoral de certaines zones: le tout nouveau département de Ranérou (Ferlo), qui dessine une large plage bleue sur la carte, compte à peine plus de 10000 inscrits, celui de Kédougou moins de 40 000. Il n'empêche. Longtemps présentés comme des bastions du PS ${ }^{13}$, ces espaces non Wolof, parmi les plus pauvres et les moins urbanisés du pays, ont changé de camp. L'homogénéité d'un vote rural sensible aux consignes des autorités - c'est sans doute dans ces périphéries que le pouvoir de l'administration territoriale est resté le plus fort malgré la décentralisation - peut constituer un facteur explicatif. La peur d'être « punis » par le régime a aussi fonctionné : le pouvoir a joué sur l'argumentation selon laquelle ses réalisations avaient été moindres dans ces régions parce qu'elles s'étaient situées dans le camp de l'opposition. Pour bénéficier de ses nombreux projets, il faudrait voter pour celui qui a les moyens de les mettre en oeuvre, c'est-à-dire le président. De la même façon, la vallée du fleuve Sénégal bascule dans le camp présidentiel. Du département de Kanel $(64,90 \%)$ à celui de Podor (65,50\%), toute la moyenne vallée Haalpular vote Wade à près des deux tiers.

Ses scores les moins élevés proviennent de certains fiefs de leaders de l'opposition, mais aussi des départements les plus urbanisés, où la dispersion des voix est plus grande qu'en milieu rural. A Dakar, Saint Louis, Kaolack, Wade obtient «seulement» autour de $50 \%$ des voix.

Le vote pour les autres candidats a donc été extrêmement faible. La multiplication des candidatures n'a rien changé sur l'échiquier : les 7 candidats qui tentaient d'entrer sur la scène politique totalisent ensemble moins de $3 \%$ des suffrages. Les quatre premiers, connus pour avoir exercé de hautes responsabilités gouvernementales ou pour diriger les partis les plus importants ${ }^{14}$ (Abdoulaye Wade, Idrissa Seck, Ousmane Tanor Dieng, Moustapha Niasse) concentrent $90 \%$ des votes. Les trois opposants les mieux classés recueillent près de $80 \%$ des voix de l'opposition. Celles-ci se sédimentent autour de quelques bassins régionaux, qui sont appelés à jouer un rôle croissant dans la vie politique nationale, à l'heure où la décentralisation confère davantage de pouvoir aux édiles locaux et où la perspective de la succession d'Abdoulaye Wade confère une valeur nouvelle au contrôle des municipalités.

Idrissa Seck apparaît comme le gagnant du camp des perdants. Après sa disgrâce de 2004 et son séjour en prison de 2005, il se classe au second rang de l'élection (14,93\%), ce qui lui permet de se placer en position avantageuse dans la course à la succession du «Vieux ». Il est le seul à battre le président sortant, dans son fief de Thiès - avec $55 \%$ des voix -, qui confirme son image de ville frondeuse vis-à-vis du pouvoir de Dakar. Il réalise 
quelques scores honorables près de Thiès (29\% à Tivaouane), à Dakar et dans ses populeuses banlieues (Pikine, Guediawaye, Rufisque), à Saint Louis, mais aussi dans quelques départements du Nord marqués par l'émigration comme Louga, où il devance le candidat du PS, avec des scores compris entre 17 et $23 \%$. Outre son bon enracinement local autour de Thiès, il doit ses scores à ses talents de communicateur - qui lui dessinent une image de compétence et de conviction qui contraste dans le paysage politique actuel - et à l'ambiguïté de son parcours, qui le fait s'afficher comme héritier légitime du sopi, tout en s'opposant au président (cf. photo 1).

Photo 1 : Idrissa Seck, « le candidat du vrai changement » ? (Cliché : G. Magrin)

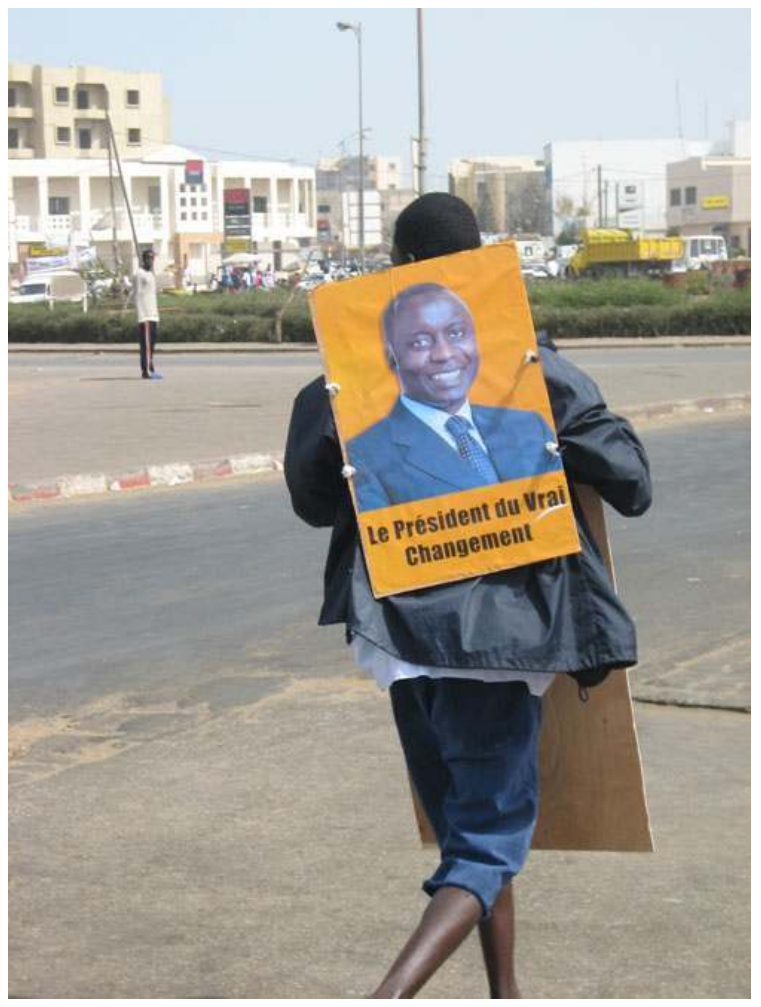

En bon communicateur, Idrissa Seck joue sur les symboles : il choisit l'orange des révolutions pacifiques d'Europe de l'Est, essaie de récupérer à la fois l'héritage du sopi et le besoin de changement. Il est le seul à contrebalancer l'omniprésence du président Wade dans l'affichage électoral. Y compris par des moyens originaux...

Ousmane Tanor Dieng (13,57 \%) obtient un score national proche de celui d'Idrissa Seck. Mais sa faiblesse, la troisième position et la déroute enregistrée dans certains bastions traditionnels sanctionnent la perte d'influence de l'ancien parti dominant. Le PS réalise une performance honorable à Mbour (33\%). Ailleurs, ses meilleurs résultats (entre 15 et 22 \%) sont enregistrés plutôt dans le Sénégal de l'Ouest, à Dakar et Rufisque, Saint-Louis, ainsi que dans les départements du bassin arachidier de Louga, Diourbel, Fatick, Nioro ou Kaffrine. La géographie du vote socialiste tient largement à ce qui a pu être préservé des départs ou des «transhumances ». Le cas de la Casamance est emblématique à cet égard. Robert Sagna, maire PS de Ziguinchor, a choisi de se présenter contre le premier secrétaire du parti. Il recueille de bons scores dans les départements casamançais (entre 25 et $28 \%$ dans les départements de Ziguinchor, Bignona, Oussouye), réduisant son rival socialiste à des scores dérisoires de 1,5 à 3\%. 
19 Moustapha Niasse est sans conteste le grand perdant de l'élection de 2007. Arrivé en troisième position en 2000 derrière le président sortant Abdou Diouf et Abdoulaye Wade, il avait pu se poser en faiseur de roi en appelant à voter pour le candidat du sopi au second tour. Avec moins de $6 \%$ des voix au niveau national, le leader de l'AFP ne sauve l'honneur que chez lui à Nioro du Rip $(36,02 \%)$, où il semble cependant devancé d'une poignée de voix par Abdoulaye Wade, ainsi que dans les départements environnants du Saloum (18,64 à Kaolack, 20,64 \% à Kaffrine). Ailleurs, il est inexistant.

3. Radiographie d'un succès

20 La conjugaison de quatre éléments principaux explique, selon nous, l'ampleur de la victoire d'Abdoulaye Wade : le temps, le vide (de l'opposition), le rêve et ... quelques réalités.

Paradoxalement, le temps a joué pour le vieux président. Opposant historique à Senghor puis au régime socialiste durant près de quarante ans, il n'aurait, en cas de défaite, exercé le pouvoir que durant 7 ans. Nombre de Sénégalais ont probablement estimé qu'après les 20 ans de pouvoir de Léopold Sédar Senghor (1960-1980) puis d'Abdou Diouf (1980-2000), Wade méritait quelques années de plus, ne serait-ce que pour terminer les nombreux chantiers entamés. Ses 80 ans ont probablement aussi rassuré : Gorgui («le vieux») est une figure paternelle, en plus d'être paternaliste. Sur un continent où l'on craint de voir les hommes politiques s'accrocher au pouvoir au-delà du raisonnable, le grand âge est une promesse de changement : ce mandat devrait être le dernier.

Le vide offert par l'opposition est une des clés principales de cette élection. Longtemps formellement unie au sein de la coalition de la CPA, l'opposition s'est divisée peu avant l'élection, où elle est partie en ordre dispersé. Mais surtout, durant les 7 ans de l'alternance, elle est apparue peu pugnace et absente, à l'image de certains de ses dirigeants, comme Moustapha Niasse, trop souvent en déplacement à l'étranger. Empêtrée dans les questions de leadership et secouée par les fréquentes «transhumances » orchestrées par Abdoulaye Wade et ses proches, elle a délégué de fait son rôle aux médias privés pour critiquer la multiplication désordonnée des projets présidentiels et une gouvernance parfois peu orthodoxe. Les stratégies personnelles poursuivies par tel ou tel chef ont conforté l'image d'une opposition visant avant tout à positionner ses responsables dans un futur gouvernement. La candidature de Landing Savané, ministre du gouvernement en poste qui se présente face à Abdoulaye Wade, était illisible pour les électeurs. Il ne recueille que $2 \%$ des voix. Il est fort possible que le socialiste Robert Sagna, en se présentant contre le candidat de son parti, ait préparé les conditions de son entrée dans un prochain gouvernement PDS.

Idrissa Seck présente un cas un peu particulier. Longtemps très proche de Wade, aux côtés duquel il a contribué à construire l'appareil du PDS et permis sa victoire, il a probablement bénéficié dans l'opinion de sa disgrâce et de son incarcération, aux bases juridiques fragiles. Durant ses 6 mois d'emprisonnement, il a montré sa force de caractère et a ainsi acquis une stature de successeur possible du président. Cela étant, ses entrevues répétées avec celui-ci en janvier 2007, nimbées de mystère, n'ont guère accrédité la sincérité de son ancrage dans l'opposition. Face à cette opposition évanescente et peu crédible, le président Wade a imposé, 7 ans durant, sa présence quotidienne dans les médias d'Etat - notamment la télévision -, donnant l'impression d'être en campagne électorale depuis les lendemains de son élection de 2000. Si le quasi-culte de la personnalité dont la couverture de ses faits et gestes procède a pu faire sourire ou agacer, il n'en demeure pas moins que Wade a su imposer sa figure familière et paternelle dans 
l'intimité de la majorité des foyers Sénégalais, au point de se positionner en quasisuccesseur de Senghor dans l'imaginaire national, escamotant la "parenthèse » Abdou Diouf (Diop M.C. 2004). Face au fourmillement de candidatures fantaisistes et à l'incertitude de la succession, Gorgui est connu et rassurant.

Et puis il fait rêver. Si les « intellectuels » sont prompts à stigmatiser l'informalisation et les dysfonctionnements de l'Etat, il est certain que le style de gouvernement d'Abdoulaye Wade séduit une bonne partie de l'opinion. Loin de l'élitisme intellectuel de Senghor ou de la rigidité technocratique d'Abdou Diouf, le populisme de Wade incarne une synthèse, efficace politiquement, entre la fierté pour l'excellence sénégalaise et le triomphe du modèle Baol Baol. Le président, qui se fait couramment appeler «maitre » en référence à son passé d'avocat, a des diplômes dans de nombreuses disciplines et intervient dans tous les domaines. Il a obtenu nombre de prix et distinctions internationales, cueillant les fruits du statut de vitrine démocratique unique de l'Afrique francophone depuis la crise ivoirienne, et propulsé le petit Sénégal, avec ses 11 millions d'habitants, dans le carré des promoteurs du NEPAD, composé par ailleurs du géant nigérian, de la riche Afrique du Sud et de l'Algérie pétrolière. Parallèlement, son charisme, ses pratiques de gouvernement et ses liens revendiqués avec le mouridisme inscrivent sa présidence sous le signe de la «baolbaolisation » de l'Etat, en miroir de celle de la société sénégalaise (voir Diop, 2004) ${ }^{15}$. Les distances avec l'éthique et la transparence ne se traduisent pas par un rejet significatif en terme de vote ${ }^{16}$.

Il y a aussi autre chose, en lien plus direct avec la campagne électoral. Tout le monde a beaucoup promis. Et le président autant, et peut-être plus que les autres. La profusion de ses projets et promesses, en forme d'inventaire à la Prévert (Michel, 2007b), n'a pas rebuté l'électorat. Les Sénégalais ne sont pourtant pas dupes, et savent l'écart des projets présidentiels aux réalités. Mais, comme le résume Madiambal Diagne, directeur du journal Le Quotidien (cité par Bernard, 2007a) « il joue sur les fantasmes d'une population très pauvre qui a tellement envie d'entendre ce qu'il raconte ".

Une de ses affiches de campagne résume bien ce plaisir à rêver d'un avenir meilleur (cf. photo 2). Mais le «meilleur à venir " est ambigu. La pêche moderne est surtout contrôlé par des armateurs étrangers, et doit faire face à la réduction des ressources. Les bassins de rétention, encore peu nombreux par rapport aux intentions, ne sont pas une panacée face aux problèmes de l'agriculture ${ }^{17}$. Quant au modèle agricole proposé sur les photos du bas - des champs de blé beauceron récoltés à la moissonneuse batteuse, un élevage intensif hollandais, allemand ou breton - il témoigne de la force des représentations européennes dans l'imaginaire sénégalais. Il reprend aussi certaines lubies présidentielles, comme celle de l'inéluctable modernisation de l'agriculture et de l'élevage sur le modèle intensif et mécanisé. Les concertations autour de la loi d'orientation agro-sylvo-pastorale adoptée en juin 2004, qui avaient montré l'attachement des ruraux à l'exploitation familiale et celui des éleveurs à l'élevage pastoral extensif ${ }^{18}$, sont de peu de poids face aux séductions de modèles technologiques étrangers, à l'honneur dans le Plan REVA ${ }^{19}$. Ce type de rêve techniciste et moderniste du développement n'est pas sans rappeler les illusions des années 1960. Sopi? 
Photo 2 : Le rêve agricole sénégalais (Cliché : C. Burger)

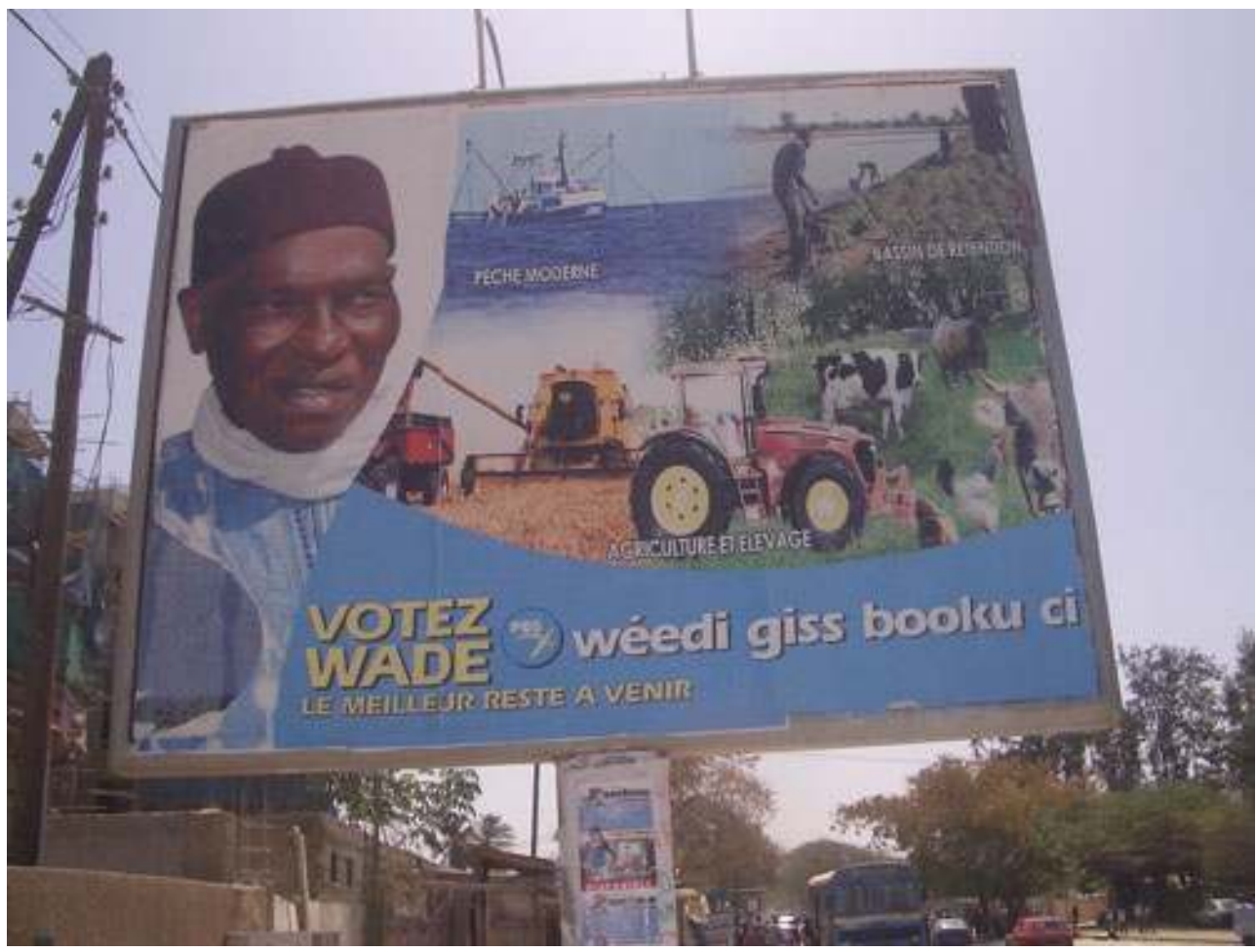

Mirage d'un développement à l'occidental évoquant les illusions des années 1960, ou ambition réaliste pour un Sénégal émergent?

Mais le succès du président Wade s'explique aussi par quelques réalités favorables. La situation économique du Sénégal n'est pas si mauvaise: malgré les difficultés de l'agriculture ou de certaines branches de l'industrie, l'argent circule dans le pays. Les taux de croissance en témoignent, toujours supérieurs à $5 \%$ de 2000 à 2005, avant de fléchir en 2006. Cette circulation monétaire est alimentée d'abord par l'aide extérieure une des plus élevée du monde par habitant - cette rente apparaissant comme le prix de l'entretien de la "vitrine » sénégalaise (OCDE, 2005). Son origine est très diversifiée : habile en la matière et servi par un président voyageur, le régime a su capter les largesses des grandes agences d'aide d'obédience occidentale (Banque mondiale, Union européenne, PNUD, coopérations bi-latérales de la France, des Etats-Unis, du Japon, etc.), mais aussi celles du monde arabe ${ }^{20}$ ou des Chines ${ }^{21}$ - ces dernières se caractérisant par une certaine opacité de nature à élargir les marges de manœuvre politiques du régime.

Les transferts des émigrés contribuent aussi pour beaucoup à lubrifier les rouages $d u$ système social sénégalais ${ }^{22}$. Avec des investissements d'origine non identifiés blanchiment d'argent international ? Epargne de sécurité d'élites africaines soucieuses de diversifier géographiquement leur patrimoine ? - ils contribuent à nourrir un rythme de construction échevelé à Dakar et dans certaines autres villes, soutenant l'emploi.

Des réformes bien menées dans le domaine fiscal, à travers une meilleure maîtrise de la TVA, garnissent les caisses de l'Etat (OCDE, 2005). Cela a permis au gouvernement d'accorder des bourses à tous les étudiants, puis, quelques mois avant les élections, d'instaurer la gratuité des soins aux personnes âgées, de recruter dans la fonction publique, d'augmenter sensiblement les salaires de plusieurs catégories de fonctionnaires. La machine redistributrice de l'Etat semble se remettre à fonctionner, 
comme si l'ajustement structurel n'était plus qu'un mauvais souvenir. D'ailleurs, l'Etat investit aussi. A côté d'un certain nombre de réalisations discutables (cf. supra), le rythme de construction des collèges et des lycées atteint un niveau jamais connu auparavant. Les grands travaux de Dakar, dont les premiers tronçons de route sont opportunément inaugurés quelques jours avant les élections, répondent à des besoins évidents de fluidité urbaine. De mémoire de Dakarois, la ville n'avait jamais connu semblables chantiers.

Enfin, plus prosaïquement, cette relative aisance de l'Etat s'est traduite par une large utilisation au service de la campagne électorale du parti au pouvoir. Les moyens de l'administration ont été utilisés. Mais surtout, de l'argent a été distribué. Ces pratiques sont anciennes au Sénégal. Mais, là encore, il semble qu'on ait cette année atteint des niveaux inégalés (voir Diakhaté D., 2007), tant au profit des simples citoyens assistant aux meetings que des "porteurs de voix" - chefs religieux notamment. Les ndigal ${ }^{23}$ de quelques marabouts secondaires très influents chez les jeunes des grandes villes - comme Cheikh Béthio Thioune ou Sérigne Modou Kara - appelant à voter pour le président Wade ont sans doute contribué à amplifier encore son succès.

Conclusion

31 La victoire électorale de grande ampleur du président Wade aux élections du 25 février 2007 ne souffre guère de contestation. En très fin politique, Abdoulaye Wade a su canaliser à son profit une conjoncture politique et économique qui aurait pu le mettre en difficulté. Autrement dit, son génie politique lui a permis de porter à son crédit aussi bien ses réalisations que ses promesses non tenues. Une des forces du sopi, slogan circonstanciel plus que véritable projet politique, était d'avoir porté des espoirs hétéroclites de changement. Ceux qui attendaient la consolidation des institutions, de la démocratie et la transparence dans la gouvernance des affaires publiques auront sans doute été les plus déçus. Mais ils n'auront pas été les plus nombreux. Sans qu'on puisse tenir que cette dimension soit indifférente aux Sénégalais, elle n'a pas cristallisé de ligne d'expression politique. Tant que le système redistributif fonctionne, le pouvoir reste légitime.

Face aux tensions que traverse la société sénégalaise - qui doit gérer une croissance démographique et une urbanisation très rapide, ainsi que l'équilibre entre des influences culturelles occidentales, arabo-musulmanes et héritées - choisir le candidat du sopi, c'était opter pour la continuité et renvoyer à un plus tard indéfini la période de l'après Wade, lourde d'incertitudes. Que tout change pour que rien ne change, dans l'immédiat. La succession du président Wade verra la fin de la génération politique des années 1960. Les conditions seront-elles alors réunies pour un véritable changement du système politique caractérisé jusque là par le clientélisme et les liens politico-religieux, dans un sens plus favorable au développement?

\section{BIBLIOGRAPHIE}

Bernard Ph., 2007a. Abdoulaye Wade confronté à un Sénégal désenchanté, Le Monde, 25 février 2007. 
Bernard Ph., 2007b. « Une économie fragilisée par la crise de l'agriculture et de l'industrie », Le Monde, 25 février 2007.

Coulibaly A., 2003. Wade, un opposant au pouvoir. L'alternance piégée ?, Dakar, Les éditions sentinelles, $300 \mathrm{p}$.

Dahou T., Foucher V., 2004. Le Sénégal, entre changement politique et révolution passive. Politique africaine $\mathrm{n}^{\circ}$ 96, Sénégal 2000-2004, l'alternance et ses contradictions, p. 5-21.

Diakhaté D., 2007. « Entretien avec le Professeur Djiby Dikhaté », Le Populaire, jeudi $1^{\mathrm{er}}$ mars 2007.

Diop M.C., 2004. Essai sur « l'art de gouverner » au Sénégal. In Diop M.C. (dir.), Gouverner le Sénégal. Entre ajustement structurel et développement durable. Paris, Karthala, p. 9-39.

Havard J.F., 2004. De la victoire du « sopi » à la tentation du « nopi ». « Gouvernement de l'alternance » et liberté d'expression des médias au Sénégal, Politique africaine $n^{\circ} 96$, Sénégal 2000-2004, l'alternance et ses contradictions, p. 22-38.

Michel S., 2007a. Au Sénégal, Abdoulaye Wade n'envoûte plus qu'une partie des marabouts de Touba., Le Monde, 24 février 2007.

Michel S., 2007b. Les ombres d'Abdoulaye Wade. Le Monde, 25 février 2007.

OCDE, 2005. Sénégal 2003-2005, 16 p.

Tall M. J.-C., 2005. « Les chantiers de Thiès » ou réflexion sur les marchés publics, Walfadjiri, vendredi 9 décembre 2005.

\section{NOTES}

1. Changement en wolof. Slogan d'Abdoulaye Wade lors de son élection en 2000.

L'expression « sopi or not sopi » forme le sous-titre du texte de Dahou et Foucher 2004.

2. Les chiffres utilisés sont issus des résultats provisoires de la Commission nationale de recensement des votes.

3. Voir Bernard Ph. 2007a.

4. En 2006 , le Sénégal est classé au $156^{\mathrm{e}}$ rang sur 177 de l'indicateur du développement humain du PNUD, en légère régression par rapport à 2000 ( $\left.154^{\mathrm{e}} \mathrm{rang}\right)$.

5. On pense ici aux scrutins dont les résultats n'ont pas été dénaturés par les fraudes.

6. Initialement programmées en même temps que les présidentielles, des législatives devraient suivre en juin.

7. Voir décret n²006-1333 du 27/11/2006.

8. Anciens cadres du Parti socialiste passés au PDS à la faveur de l'alternance.

9. L'utilisation des grands travaux publics pour alimenter le système clientéliste qui cimente le pouvoir est une constante de la vie politique sénégalaise depuis l'indépendance. Les chantiers de la Corniche financés par l'OCI dans des conditions de transparence très relative n'échappent pas à cette règle. Voir M.J.C Tall, 2005.

10. Il s'agit d'aménager à Diamnadio, à l'intersection des routes Dakar Thiès et Dakar Mbour (qui mènera au nouvel aéroport de Diass), une plate-forme destinée à accueillir des industries modernes, à côté de lotissements nouveaux, et de la relier à la capitale par une autoroute à péage.

11. L'Agropôle de Rao en constitue un bon exemple : situé au débouché de la zone maraîchère du Gandiolais, au sud de Saint Louis, ce centre de stockage et de conditionnement moderne des produits agricoles, réalisé sur financement espagnol sans 
préparation suffisante, a été terminé en avril 2005. En mars 2007, il n'avait toujours connu aucune activité.

12. En 2005 et 2006, des dizaines de milliers de migrants clandestins sub-sahariens ont abordé les côtes canariennes, au prix de nombreuses pertes humaines - non quantifiées. Une forte proportion d'entre eux venait du Sénégal.

13. La région de Tambacounda était restée au PS lors des élections locales de 2002, comme environ la moitié des communautés rurales de la Vallée.

14. Moustapha Niasse (AFP) et Idrissa Seck (dissident du PDS) ont tous deux été premier ministre. Ousmane Tanor Dieng est le premier secrétaire du PS.

15. Le modèle Baol Baol, qui déborde de la région éponyme pour englober l'ouest et le nord-ouest du bassin arachidier, s'enracine dans des régions à peuplement Wolof, sous influence mouride. Il est caractérisé par la pratique généralisée du commerce, le faible niveau d'éducation, le règne de l'informel dans tous les domaines, une forte émigration internationale (principalement vers l'Italie, les Etats-Unis, plus récemment l'Espagne), la débrouillardise (parfois jusqu'aux marges de la légalité...).

16. Le parallèle avec la popularité de Jacques Chirac est tentant. On pardonne au président d'avoir pris quelques libertés avec la loi, car les citoyens le font aussi, et cela le fait paraitre d'autant plus proche d'eux.

17. On peut citer la durabilité agronomique et les tensions foncières, l'accès au crédit, les difficultés de commercialisation, etc.

18. Auxquelles on peut associer les avis scientifiques dominants insistant sur l'adaptation de ce type d'élevage aux contraintes écologiques et économiques sahéliennes.

19. REVA : retour vers l'agriculture. Il vise à financer des pôles de modernisation agricole destinés aux jeunes, notamment des citadins sans emploi. Annoncé en 2006 sans lien clair avec la LOASP qui venait d'être votée, ce plan concilie l'émission d'un signal à destination des Européens face à l'émigration massive (tout en justifiant l'aide), la réalisation d'ambitions présidentielles anciennes (modernisation) et des préoccupations électorales (clientélisme).

20. L'Organisation de la conférence islamique finance actuellement les grands travaux routiers d'aménagement de la Corniche à Dakar.

21. Taïwan a notamment financé les débuts du spectaculaire programme de construction des Cases des tout petits, avant que le Sénégal ne décide, en 2005, de « changer de Chine » au profit de la République populaire.

22. Il y aurait entre 1,5 et 3 millions de Sénégalais à l'extérieur du Sénégal. Leurs transferts, qui atteindraient 300 à 500 millions d'euros par an, représenteraient 5 à $10 \%$ du PIB (Dahou, Foucher, 2004), et seraient en augmentation (OCDE, 2005).

23. Ordre donné par le marabout, qui cimente son lien avec ses disciples.

\section{RÉSUMÉS}

La large victoire au premier tour du sortant A. Wade aux élections présidentielles sénégalaises du 25 février 2007 a surpris : la géographie électorale ne laisse que de rares îlots à l'opposition. Certaines difficultés économiques et politiques alimentaient bien le mécontentement populaire. 
Mais l'inefficacité de l'opposition, l'habileté du président, des éléments de conjoncture favorable et l'incertitude de l'après Wade ont finalement amené nombre de Sénégalais à choisir le sopi (changement) par la continuité.

The large victory of the going out president A. Wade at the first round of the Senegalese presidential elections of February 25th 2007 has surprised : the electoral geography only leaves few pockets to the opposition. Some economic and political difficulties fed the dissatisfaction of the people. But the opposition inefficiency, the President cleverness, some favourable climate elements and the worries about the "after Wade" at last brought a lot of Senegaleses to choose the sopi (change) by continuity.

\section{INDEX}

Keywords : Senegal, presidential elections, electoral geography

Mots-clés : Abdoulaye Wade, élections présidentielles, géographie électorale

\section{AUTEUR}

\section{GÉRAUD MAGRIN}

Géraud Magrin magrin@cirad.fr, Université Gaston Berger de Saint Louis, chercheur au CIRAD (département environnements et sociétés, UMR TETIS)

Ses deux publications les plus significatives sont:

- Magrin G., 2001. Le Sud du Tchad en mutation. Des champs de coton aux sirènes de l'or noir. Paris, Sépia-Cirad, 427p.

- Magrin G., 2006. «La décentralisation résoudra les problèmes de l'Etat en Afrique », Courade G. (dir.), L’Afrique des idées reçues, Paris, Belin : 383-389. 